\title{
A Study to Determine Knowledge, Attitude and Practices of Women of Child Bearing Age on Obesity and Its Impact on Fertility at Mbuyanehanda Maternity Hospital
}

\author{
Article by Steadyfaith Mataga \\ Textile American University, Guyana, South America \\ E-mail id: faithmataga@gmail.com
}

\begin{abstract}
Background: Shortage of obesity awareness campaigns at antenatal clinics; women who were severely obese were $43 \%$ less likely to achieve pregnancy than normal-weight women or women who were considered overweight but not obese during the study. The study is among the first to examine the relationship between body weight and infertility in women who ovulate in Harare Zimbabwe. In Harare only five out of twentytwo antenatal clinics had obesity health education talks and measured BMI for ovulating women. In 2018 this prompted an investigation to determine knowledge, attitude and practices of women of child bearing age on obesity and its impact on fertility at Mbuyanehanda Maternity Hospital.

Methods: Analytical cross-sectional study was carried out. Three hundred and eighty-four (384) participants were selected by proportional sampling from Mbuyanehanda antenatal clinic. Questionnaires adapted from the World Health Organization Stepwise approach to Surveillance noncommunicable disease instrument and the Health Belief model was used to collect data on knowledge, attitude and risk factors of obesity on fertility in women of childbearing age. BMI measurements were taken. Obesity was defined as BMI above 25.

Results: The proportion of patients who were obese and had their BMI measured was 70\%. Only females were enrolled in the study. The statistically significant factors associated with obesity were having heard about the risk obesity (AOR 3.1, 95\% C.I 1.7-5.4), knowing that weight check and control was important whilst on childbearing age (AOR 1.8, 95\% C.I 1.1-2.9) and health education on dangers of obesity or high BMI (AOR $1.695 \%$ C.I 1.0-2.7).

Summary: There was a high proportion of elevated BMI amongst patients who were ignorant of the dangers of obesity on fertility. Despite lack of knowledge on BMI monitoring equipment and shortage of staff, majority of patients at Mbuyanehanda maternity hospital of childbearing age were at risk of developing infertility or childbearing complications because of inadequate knowledge on obesity prevention and control. Health promotion and education on dangers of obesity and body weight monitoring and the upgrading of health services factors to improve the monitoring of BMI at maternal health clinics is necessary. Further studies on factors associated with poor control of body weight amongst female patients during child bearing age are needed.
\end{abstract}

Keywords: Body Mass Index (BMI), fertility, Health Belief Model, Obesity.

\section{Abbreviations}

WOCBA : Women of child bearing Age

BMI : Body Mass Index

WHO : World Health Organization

MNMH : Mbuyanehanda Maternity Hospital

HBM : Health Belief Model 


\section{Introduction}

The prevalence of overweight and obesity is higher among urban women than their rural counterparts in Zimbabwe. More than 33\% of women in the urban area are either overweight or obese compared to only $11 \%$ of women in rural areas according to the Maternal reproductive health survey conducted by Ministry of health and Child care in 2012. Obesity is a global issue. Despite efforts to confront it, the worldwide incidence of obesity continues to escalate. The WHO estimates that approximately one billion people throughout the world are overweight and that over 300 million of these are obese. If current trends continue, the number of overweight persons will increase to 1.5 billion by the year 2015 . The number of obese adults in Australia is estimated to have risen from 2 million in 1992 to 3.1 million in 2005 [2]. What contributes to the problem of obesity mainly stems from the imbalance between reduced exercise, overindulgence in energy-dense dietary intake, changing lifestyle and dietary composition. It has been known that obesity is related to systemic diseases such as diabetes mellitus, cardiovascular diseases, hyperlipidemia, sleep apnea, cancers and osteoarthritis. Female obesity has a great impact on reproductive function and the hormonal milieu. As far back as 1934, the association between obesity and infertility was recognized when Irving Stein and Micheal Leventhal described a triad consisting of obesity, hirsutism and infertility: the 'Stein-Leventhal Syndrome'. Overweight and obese women have more problems of menstrual irregularities, chronic or intermittent anovulation, infertility, signs of androgen excess, increased risk of miscarriages and hormone-sensitive carcinomas. In the Million Women Study, it was noted that increasing BMI was associated with an increased incidence of endometrial carcinoma (relative risk $[R R]=2.89 ; 95 \%$ CI: $2.62-3.18$ ). Obesity also has important implications for the delivery of obstetric care. It is well recognized that maternal obesity is associated with an increased risk of maternal, peripartum and neonatal complications.

It is not clear how obesity affects fertility in women who ovulate normally. Van der Steeg suggests that disruptions in the hormone leptin, which regulates appetite and energy expenditure, may prevent successful fertilization.

Reproductive endocrinologist William Dodson, MD, tells WebMD that it is increasingly clear that the role of obesity in reproduction is more complex than was once thought ${ }^{1}$.

"What we once held as dogma is now starting to fall apart," he says. "We thought that if a woman's obesity was not affecting her ovulatory function, her fertility would be similar to a normal-weight woman's. But this does not appear to be true."

Dodson's own recent research at the Penn State Hershey College of Medicine confirmed that obese women undergoing infertility treatments needed higher doses of infertility drugs than normal-weight or overweight women.

Like the newly published study, all the women in the Penn State study had normal ovarian function.

"The issue of obesity and reproduction is complex, and we are only beginning to understand it," he says. Obesity contributes significantly to menstrual irregularities, absence of ovulation, difficulty in conception, reduced response to fertility treatments, increased chances of miscarriage and perinatal complications. Obesity causes increased production of insulin as well as insulin resistance, which, in women, may lead to erratic ovulation. There is also a link between obesity, excess insulin production and infertility which is referred to as polycystic ovarian syndrome (PCOS). PCOS is a specific medical condition represented with irregular menstrual cycles, decreased or absence of ovulation, obesity and elevated levels of male hormones. PCOS is a risk factor for infertility, and it has been established that 35-65\% of PCOS patients are obese. Increased BMI negatively affects the achievement and maintenance of pregnancy. Obese females have a lower chance of pregnancy and a higher risk of miscarriage. Even after conception, they have higher risk of pregnancy complications such as miscarriage, developing diabetes and chances of premature birth. The likelihood of LSCS (Lower segment Caesarian section) also increases which puts the mother and baby at high risk. The prevalence of obesity among women of child-bearing age has increased from about $24.2 \%$ in 2005 to $28.3 \%$ in 2015 , and the number of females having weight-loss surgery is rising, which increases their chances of conception due to weight loss.

\section{Problem statement}

An observation has been made that women of child bearing age seem not to be aware that being obese has bearing on fertility. Nationally representative surveys show recent increases in the prevalence of 
overweight and obesity among women of reproductive age in Zimbabwe, and Zambia from 15.8 to $28.8 \%$ from 2006 to 2010 .

\section{Purpose of the study}

The purpose of the study is to determine knowledge, attitude and practices of women of child bearing age on obesity and its impact on health at Mbuyanehanda Maternity Hospital.

\section{Objectives}

The study seeks to:

- Determine knowledge of women of child bearing on obesity and its impact on fertility.

- Find out about the women's attitude and practices

- Identify challenges these women face regarding obesity and its impact on fertility.

\section{Inclusion and exclusion criteria into the study}

As measured by the BMI, a 5-foot 6-inch woman who weighs $52 \mathrm{kgs}$ to $70 \mathrm{kilograms}$ is considered normal weight (BMI of 18.5 to 24.9). If she weighs between 70 to 84 kilograms, she is considered overweight (BMI of 25 to 29.9), and she would be considered obese at a weight of 84kilograms or more (BMI of 30+).

\section{Questions of the study}

What knowledge and practices do these women have on obesity and its effects on fertility?

\section{Significance of the study}

The researcher hopes that:

- Knowledge on obesity and its impact on fertility will be determined.

- Recommendations made will be handed over to the Ministry of Health and Child Care for intervention purposes.

- Challenges identified by the women will be identified.

- More information will be given to women regarding obesity and its effects on fertility.

\section{Scope of the study}

The study will be conducted between November and December 2018 at MNMH in Harare Zimbabwe. A total of over 300 women will be interviewed. These will be from all over Zimbabwe since MNMH is a national referral centre for maternal specialty.

\section{Literature review}

According to the World Health Organization (WHO 2018) Overweight and obesity are defined as abnormal or excessive fat accumulation that presents a risk to health. A crude population measure of obesity is the body mass index (BMI), a person's weight (in kilograms) divided by the square of his or her height (in metres). A person with a BMI of 30 or more is generally considered obese. A person with a BMI equal to or more than 25 is considered overweight.

Obesity and overweight prevalence are increasing and it has become an epidemic and a public health issue of concern worldwide. Obesity has damaging effects on all systems, including reproductive health. There is a high prevalence of infertility in women with obesity. It is now well known that there is a strong association between obesity and infertility among women of child bearing age worldwide. However, studies are still ongoing on the relationship between obesity and reproductive functions. Obesity is associated with early onset of puberty, abnormal menstrual pattern, decreased efficacy of contraceptives, ovulation disorders, and increased miscarriage rate. The women tend to have poor reproductive outcomes as much as they will also have assisted conception.

\section{Research methodology}

\section{Introduction}

The chapter will describe the methods which are going to be used in carrying out the research study. It includes the research design, study population, study sample, data collection, sample procedure, 
ISSN: 2520-3134

instrument design, data analysis, and ethical considerations.

\section{Research design}

A descriptive research design is going to be used. It is appropriate for this study because it gives the researcher to first-hand information regarding knowledge, attitude and practices of WOCBA at Mbuyanehanda Maternity Hospital (MNMH)

\section{Study population}

At total of 150 (WOCBA) Women of Child Bearing Age with a body mass index of 30 and above will be randomly selected. Another 150 WOCBA with a body mass index of less than 30 will also be randomly selected.

\section{Study sample}

As it is not possible to study every woman, simple random sampling method is going to be used. Simple random sampling is going to be used so that each individual is chosen entirely by chance and each member of the target population has an equal chance of being included in the sample.

\section{Data collection instrument}

Data will be collected from 300 WOCBA on their knowledge, attitude and practices towards obesity and its effects on fertility.

\section{Sample procedure}

This is going to be done on 300 WOCBA of which 150 will be overweight and 150 who are not. Probability sampling will be used so that every possible sample of a given size will have the chance of being selected.

\section{Instrument design}

An interview schedule is going to be used to collect data.

\section{Conceptual framework}

Risk factors for BMI monitoring in childbearing mothers can be associated with demographic factors, socio-cultural factors, health service factors, economic and cultural factors (see figure 1 below). 


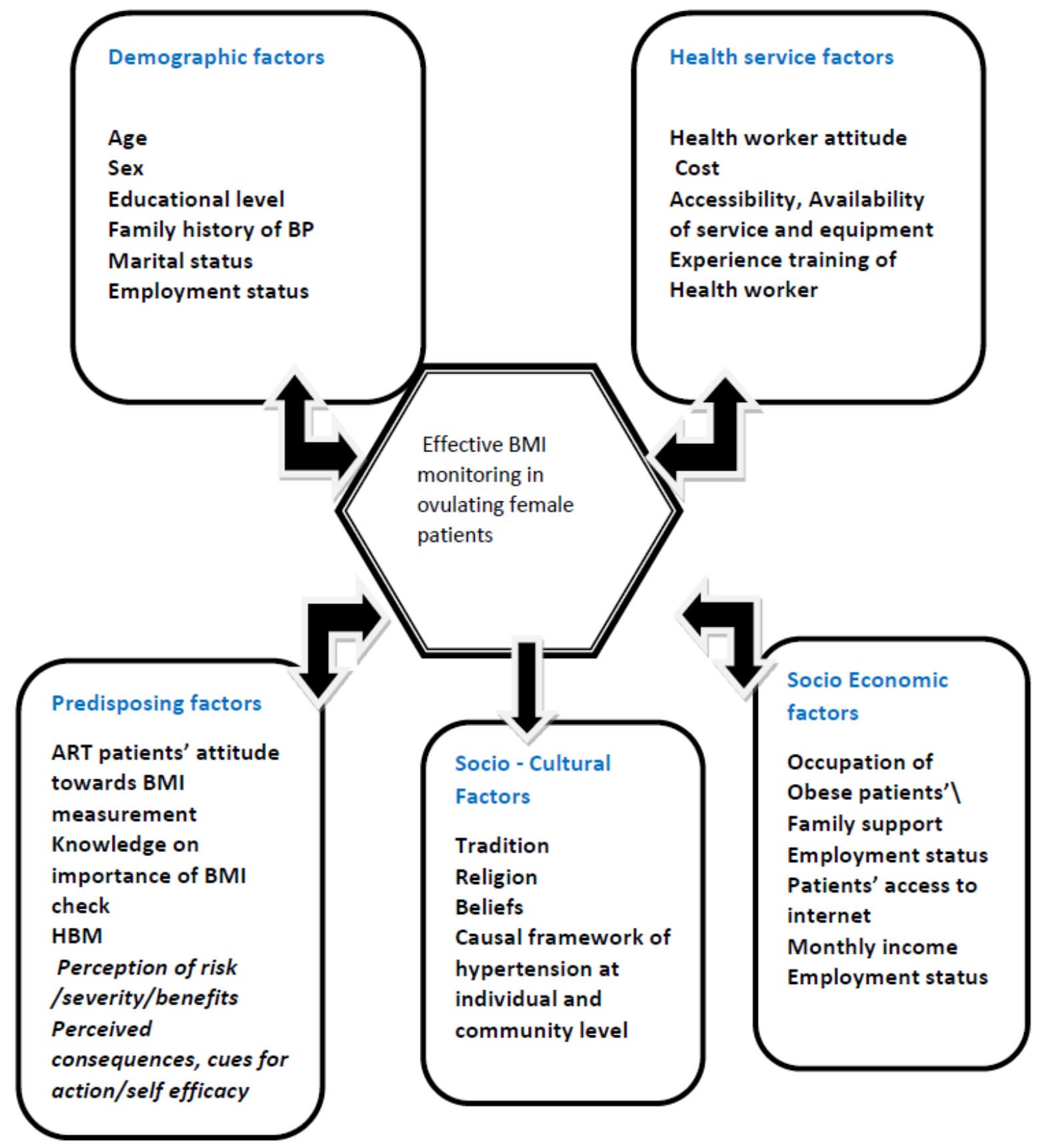

Figure 1. Conceptual framework

The health care environment should be having low barriers and high perceived benefits according to the health belief model these are important in order for there to be recommended behaviours. Incorporating the perceived benefits of action i.e. BMI monitoring and the barriers to action which may be health service factors or linked to obese patients' attitude towards BMI check, the study also assessed whether health care factors promoted prevention of infertility to patients with high BMI above 30 through monitoring and information dissemination.

Self-efficacy the need for patients with high BMI to feel competent to prevent obesity as an adverse effect of life saving low BMI is also another aspect of the HBM that the study explored. The study from the conceptual framework mainly looked at the health service factors that contributed to poor BP monitoring and detection of high BMI in patients' women of childbearing age. Predisposing factors linked to the Health belief model were assessed as well as socio economic factors and demographic factors to 
try and understand and come up with reasons why most high morbidly obese patients did not have their BMI monitored at antenatal clinics. These were included in the framework bearing in mind that in most countries BMI monitoring is now being done at home if the patients can calculate correctly their BMI as taught. At the end of the interviewer administered questioning the patients had a health talk session about risks of high body mass index and how to control or prevent its rapid effects. In addition, the study also assessed low barriers to BMI monitoring both socially economically and health related, perceived benefits and the level of knowledge of childbearing female patients with high BMI on the importance of BMI regular check.

\section{Data presentation and analysis}

The association between obesity and menstrual irregularities has been recognized. Obesity was present at a fourfold higher rate in women with menstrual irregularities compared with normally menstruating females. Historical data from 26,638 women, aged 20-40 years, were used to study the association between obesity and menstrual abnormalities, including evidence of infertility. It was found that women with evidence of anovulatory cycles, in other words, irregular cycles greater than 36 days and hirsutism, were more than $30 \mathrm{lb}(13.6 \mathrm{~kg})$ heavier than women with no menstrual abnormalities after adjusting for height and age. In adolescents and young women, the age of onset of obesity and that of menstrual irregularities are significantly correlated. In the 1958 British birth cohort, Lake et al. tracked 5799 females at the ages $7,11,16,23$ and 33 years and noted that obesity in childhood and early twenties increased the risk of menstrual problems. Obesity at 23 years and obesity at 7 years both independently increased the risk of menstrual problems by age 33 years (odds ratio $[\mathrm{OR}]=1.75$; OR $=1.59$, respectively) after adjusting for other confounding factors. Women who were overweight and obese at 23 years of age were less likely to conceive within 12 months of unprotected intercourse after adjustment for confounders (RR $=0.69$ ). Overweight and obesity in early adulthood appears to increase the risk of menstrual problems and sub fertility. There are also several epidemiological studies that suggest changes in bodyweight are critical factors regulating pubertal development in young women. A few studies have reported that obese girls had their menarche earlier than normal-weight girls. Similarly, the onset of ovarian failure and menopause occurs several years earlier in obese women than in normal-weight women [21]. The link between obesity and infertility is complex. The available knowledge supports the concept that androgen alterations and their balance with estrogen represent the most important mechanism responsible for the development of subfertility or infertility in obese women. Sex hormone binding globulin (SHBG) is a protein carrier that binds testosterone and dihydrotestosterone with high affinity and estrogens with a lower affinity. The degree of obesity is inversely related to SHBG levels. In addition, body fat distribution further influences SHBG concentrations. Females with central adiposity have lower SHBG concentrations in comparison with peripheral obesity [22]; therefore, the percentage of free testosterone fraction tends to be higher in women with central obesity. In addition, the adipose tissue is a site of active androgen production, converting androgens into estrogens, and of androgen and estrogen interconversion, which largely depends on the amount of fat [22]. Increased androgen production and reduced binding of androgens to SHBG contribute to hyperandrogenism, resulting in anovulation through inhibition of follicular maturation. Hormones regulating energy metabolism have been shown to exert different effects on several reproductive events. The roles of insulin and insulin-like growth factors have been studied extensively and are well known for their effects on reproductive events, such as ovarian steroidogenesis, folliculogenesis and ovulation physiology. Insulin resistance plays a key pathogenic role in PCOS, and insulin-sensitizing drugs are now widely used to induce ovulation and improve fertility in these women [23]. In addition to insulin, novel hormones, such as leptin, ghrelin, adiponectin, resistin and peptide YY3-36 have been discovered as important regulators of appetite and energy homeostasis [24, 25]. The close relationship between energy metabolism, nutritional state and reproductive physiology suggests that disorders or alterations in nutritional state (obesity, malnutrition, anorexia nervosa and so on) and metabolic disturbances can disrupt the complex interplay of gonadotropins and gonadal hormones, which are essential for fertility. Recent research has demonstrated that leptin plays an integral role in the normal physiology of the reproductive system with complex interactions at all levels of the hypothalamicpituitary-gonadal axis. Observational studies have demonstrated that levels of leptin excess, deficiency or resistance can be associated with abnormal reproductive function [26]. Increased body weight and fat 
tissue substantially disrupt menstrual pattern and fertility potential. In obese women, weight loss alone improves insulin resistance and promotes fertility. In addition, expression of leptin, ghrelin and their receptors in various reproductive organs, such as the ovary, testis, endometrium, embryo and placenta, have been demonstrated to have important roles in different stages of embryo development and implantation [26,27]. The Nurses' Health Study in 1994 compared 2527 married nulliparous nurses unable to become pregnant for at least 1 year owing to ovulatory disorder with controls comprising of 46,718 married parous nurses with no history of infertility [28]. The risk of ovulatory infertility for women at different BMI levels at age 18 years was compared with that for women at 18 years whose BMI was 2021.9 (median for the cohort). Logistic regression was used to adjust for other confounding variables. Multivariate relative risks for infertility were: 1.2 (BMI: <16), 1.1 (BMI: 16-17.9),

1.0 (BMI: 18-19.9), 1.0 (BMI: 20-21.9),

1.1 (BMI: 22-23.9), 1.3 (BMI: 24-25.9),

1.7 (BMI: 26-27.9), 2.4 (BMI: 28-29.9),

2.7 (BMI: 30-31.9) and 2.7 (BMI: $\geq 32$.

These findings suggested that an elevated BMI at age 18 years, even at levels lower than those considered to be obese, is a risk factor for subsequent ovulatory infertility. More recent data from this group show that ovulatory infertility can be largely attributed to overweight and sedentary lifestyle. Recent data by Gesink Law et al. from his study on more than 7327 pregnant women enrolled in the Collaborative Perinatal Project at 12 study centers in the USA showed that fecundity was reduced for overweight (OR: 0.92; 95\% CI: 0.84, 1.01) and obese (OR: 0.82; 95\% CI: 0.72, 0.95) women compared with optimal-weight women and was more evident for obese primiparous women $(\mathrm{OR}=0.66$; $95 \% \mathrm{CI}$ : $0.49,0.89$ ).

\section{Obesity and infertility treatment}

Most studies demonstrate conclusive evidence that increasing BMI is associated with an increased requirement of drugs to induce ovulation. Large doses of clomiphene of up to $200 \mathrm{mg}$ per day were required to ensure ovulation in the heaviest women. Similar trends were also observed in doses of gonadotrophins required to induce ovulation. In a study of 335 women with WHO group 11 anovulatory infertility, it was noted that with increasing BMI, a higher threshold dose of gonadotrophins was required (odds for needing a higher dose more than 75 IU was 1.47 [95\% CI: 0.84-2.55] and 2.15 [95\% CI: 1.173.94] for overweight and obese women, respectively) and there were more days of stimulation (OR: 1.8; 95\% CI: $0.32-3.27$ and OR: 2.91 ; 95\% CI: $1.21-4.6$ ) for overweight and obese women, respectively. In another study by Fedorcsak et al. involving 2660 IVF/intracytoplasmic sperm injection (ICSI) cycles, there was a positive correlation between BMI and the total follicle-stimulating hormone (FSH) required. The higher the BMI, the more FSH is required for ovarian stimulation. Although the starting dose of FSH was not fixed in this report, the correlation between BMI and the length of stimulation suggests that the differences in FSH requirement were not caused solely by a deliberate subscription of higher FSH doses in obese women. Therefore, obesity represents a condition of resistance to agents used to stimulate ovulation. Linear association was observed between higher BMI and longer stimulation with FSH ( $<$ < 0.001), requirement for increased total doses of FSH $(\mathrm{p}<0.001)$, increased frequency of cycle cancellations owing to insufficient follicular development $(\mathrm{p}<0.001)$ and a lower number of collected oocytes $(\mathrm{p}<0.001)$. The value of obesity as a predictor of infertility treatment outcome is controversial. While some studies reported a decrease in pregnancy and implantation rates in obese women, others reported no effects of extreme body weights. Conflicting results may be related to the type of treatment, inconsistent definitions of obesity and smaller size of cohorts observed. In a study by Wang et al. on a large cohort of women seeking infertility treatment $(\mathrm{n}=3586)$, it was noted that weight had a significant correlation on the outcome of assisted reproductive technique, with a significant linear reduction in fecundity from the moderate group to the very obese group $(\mathrm{p}<0.001)$. The fecundity of the moderate group was almost $60 \%$ higher than that of the very obese group. Not only that, it was observed that bodyweight has an 'inverted U' effect on reproduction, which means that being either underweight or overweight has a deleterious effect on assisted reproductive techniques (ART) outcome. There is evidence for an increased risk of miscarriage in obese women. Lashen et al. conducted a nested case-control study in which obese (BMI: > 30kg/m2) women were compared with an age-matched control group with normal 
BMI (19-24.9 kg/m2) [39]. A total of 1644 obese and 3288 age-matched normal-weight controls with a mean age of 26.6 years (95\% CI: 26.5-26.7) were included in the study. The risk of early miscarriage was significantly higher among the obese patients (OR: 1.2; 95\% CI: 1.01-1.46; $\mathrm{p}=0.04)$. These data confirm that increasing obesity significantly reduces the chance of successful pregnancy. Obesity is also a risk factor for early miscarriage after assisted reproduction. Fedorcsak et al. noted that obesity was associated with an increased risk of early pregnancy loss occurring before 6 weeks gestation. In another retrospective study on a large cohort of women $(n=2349)$ who were pregnant following ART, the overall incidence of spontaneous abortion was $20 \%$. Compared with women with normal BMI, there was progressive increase in risk of spontaneous abortion in overweight, obese and very obese groups (OR: 1.29, [95\% CI: 1.001.66]; OR: 1.71; [95\% CI: 1.20-2.43] and OR: 2.19; [95\% CI: 1.27-3.78] in the overweight, obese and very obese, respectively). The poor reproductive performance in obese women, both in natural and assisted conception cycles, may be a result of a combination of lower implantation and pregnancy rates, higher preclinical and clinical miscarriage rates, and increased complications in pregnancy for both mother and fetus. These have been related to various endocrine and metabolic disturbances, such as effects on steroid metabolism and alterations in the secretion and action of insulin and other hormones, such as leptin, resistin, ghrelin and adiponectin, which may affect follicle growth, corpus luteum function, early embryo development, trophoblast function and endometrial receptivity. The question remains as to the mechanism by which obesity may affect fecundity in women. Is it exclusively an ovarian effect, endometrial effect or a combined effect? The oocyte donation model is unique. By restricting study to oocytes donated by normal-weight women, the effect of obesity on oocyte development was eliminated, thus permitting an assessment of the extraovarian effect of excess bodyweight on successful pregnancy outcomes. However, data regarding the effects of BMI on the oocyte donation model are conflicting. While two authors observed no association between BMI, pregnancy rate and loss rate in oocyte donation cycles $[42,43]$, others noted obesity to be an independent risk factor for miscarriage in women receiving donated oocytes [44,45]. Bellver et al., in his recent publication, studied 2656 first IVF cycles of ovum donation using good-quality embryos [36]. The recipients were divided into groups according to BMI: less than $20 \mathrm{~kg} / \mathrm{m} 2(\mathrm{n}=471), 20-24.9 \mathrm{~kg} / \mathrm{m} 2(\mathrm{n}=1613), 25-29.9 \mathrm{~kg} / \mathrm{m} 2(\mathrm{n}=450)$, and greater than or equal to $30 \mathrm{~kg} / \mathrm{m} 2(\mathrm{n}=122)$. The rates of implantation, pregnancy and miscarriage were similar among the BMI groups, although there was a negative trend when BMI increased. However, the ongoing pregnancy rates per cycle were poorer in the overweight and obese groups than in the underweight and normal groups. When the cut-off point of $25 \mathrm{~kg} / \mathrm{m} 2$ was taken into account, the ongoing pregnancy rate per cycle initiated was significantly reduced in the overweight and obese groups (from $45.5 \%$ with $25 \mathrm{~kg} / \mathrm{m} 2, \mathrm{p}=0.002)$. The OR of ongoing pregnancy per started cycle among BMI groups was 0.85 (95\% CI: $0.76-0.95, p=0.003$ ), showing a significant trend to decrease as BMI increased.

Table 1. Adjusted odds ratio for maternal, peripartum and neonatal outcomes, according to BMI. Normal

\begin{tabular}{|l|l|l|l|l|}
\hline & Normal & Overweight & Obese & MorbidlyObese \\
\hline & (BMI 20.01-25) & (BMI 25.01-30) & (BMI 30.01-40) & (BMI >40) \\
\hline Maternal outcomes & & & & \\
\hline $\begin{array}{l}\text { Hypertensive disease of } \\
\text { pregnancy }\end{array}$ & 1.00 & 1.74 & 3.00 & 4.87 \\
\hline Gestational diabetes & 1.00 & 1.78 & 2.95 & 7.44 \\
\hline Caesarean section & 1.00 & 1.50 & 2.02 & 2.54 \\
\hline Neonatal outcomes & & & & \\
\hline Stillborn & 1.00 & 1.16 & 1.19 & 0.89 \\
\hline Birth defect & 1.00 & 1.26 & 1.58 & 3.41 \\
\hline Hypoglycaemia & 1.00 & 0.78 & 2.57 & 7.14 \\
\hline $\begin{array}{l}\text { Admission to intensive } \\
\text { care }\end{array}$ & 1.00 & 0.92 & 1.25 & 2.77 \\
\hline
\end{tabular}

The findings that were found at the study that we conducted at Mbuyanehanda had findings that were similar to another large review, involving women who were booked for antenatal care in Queensland, Australia, it was noted that as BMI increases, cesarean section rates, maternal morbidity, neonatal 
morbidity, neonatal intensive care utilization and length of hospital stay all increase. There was also a doubling of birth defects from $1.9 \%$ in women with a BMI of $30-40 \mathrm{~kg} / \mathrm{m} 2$ to $4 \%$ in women with a BMI above $40 \mathrm{~kg} / \mathrm{m} 2$ (Table 3). Data from the Atlanta Birth Defects Risk Factor Surveillance Study from January 1993 until August 1997, noted that obese women were more likely than average-weight women to have an infant with spina bifida (unadjusted OR: 3.5; 95\% CI: 1.2-10.3), omphalocele (OR: 3.3; 95\% CI: 1.0-10.3), heart defects (OR: 2.0; 95\% CI: 1.2-3.4), and multiple anomalies (OR: 2.0; 95\% CI: 1.0 3.8) [50]. Overweight women were more likely than average-weight women to have infants with heart defects (OR: 2.0; 95\% CI: 1.2-3.1) and multiple anomalies (OR: 1.9; 95\% CI: 1.1-3.4). Several epidemiologic studies have suggested that being overweight before pregnancy is a risk factor for neural tube defects (NTDs) [51,52]. BMI of more than $29 \mathrm{~kg} / \mathrm{m} 2$ doubles the risk of NTDs (OR 1.9; 95\% CI: 1.1-3.4) [52]. Werler et al. compared 604 fetuses or infants with a NTD with 1658 fetuses or infants with other major malformations, all diagnosed within 6 months of delivery [53]. Compared with mothers weighing 50-59 kg, the RR of NTDs increased to 1.9 (95\% CI: 1.2-2.9) for women weighing 80-89 kg and to 4.0 (95\% CI: 1.6-9.9) for women weighing $110 \mathrm{~kg}$ or more. When women were classified according to daily intake above or below the recommended level of $400 \mu \mathrm{g}$ of folate, an approximately threefold increase in risk was estimated for the heaviest weights in both groups. Intake of $400 \mu \mathrm{g}$ or more of folate reduced the risk of NTDs by $40 \%$ among women weighing less than $70 \mathrm{~kg}$, but no risk reduction was observed among heavier women [53]. The supposed mechanisms that increase the congenital anomaly rates include insulin resistance and its consequence, incipient hyperglycemia. In addition, the increased risk of congenital malformations, in particular NTDs, in children of obese women can be explained by difficulties in visualization when making the ultrasound scan and missing adjustment for weight when measuring biochemical markers [54]. Thus, preventive measures should be taken with overweight teenagers before their first pregnancy, and delivery wards should have an essential role in identifying women at high risk in their next pregnancy as a result of obesity. Modest weight loss would bring substantial advantages to the obstetric outcomes of these women.

\section{Summary, discussion and conclusion}

Treatment of obesity itself should be the initial aim in obese infertile women before embarking on ovulation-induction drugs or ART. Reduction of fat and abdominal fat should result in improved menstrual function and fertility and a reduction of metabolic risks. A reduction of 2-5\% in body weight was associated with restoration of ovulation, an $11 \%$ reduction in abdominal fat, a $4 \mathrm{~cm}$ reduction in WC and a $71 \%$ increase in insulin sensitivity. Weight loss results in an increase in SHBG, reduction in testosterone, improved menstrual function, improvement in conception rate and reduction in miscarriage rate. As central adiposity is associated with menstrual disorders and infertility, abdominal fat loss is critical in restoring ovulation. Various strategies have been suggested to overcome the problem of obesity. Amongst these are dietary management, physical activity, behaviour modification, pharmacologic treatment and surgery. The issues are the long-term compliance to these strategies and maintaining the weight loss. The NIH recommends a multifaceted approach to treating obesity. It emphasizes the importance of achievable and sustainable goals, notably a combination of diet, physical activity and behavior therapy. This is where aspects of the health belief model came into play in terms of focusing on the perceived benefits.

\section{Obesity in women}

Obesity is related to many systemic diseases and abnormalities in hormonal and reproductive function.

Obesity can be measured clinically by body mass index (BMI), waist circumference and waist-hip ratio. Waist-hip ratio greater than 0.8 defines an increased risk of cardiovascular disease and reduce cumulative pregnancy rate.

\section{Obesity and PCOS}

$30-50 \%$ of PCOS women are overweight or obese. Obesity, particularly abdominal obesity amplifies hyperinsulinemia. 


\section{Obesity and reproduction}

Obesity is related to menstrual abnormalities; amenorrhea, oligomenorrhea and menorrhagia are fourfold higher in obese women.

Obesity also contributes to anovulatory and ovulatory infertility via altered imbalance between estrogen, androgen and SHBG.

Hyperandrogenism and hyperleptinemia are also related to anovulatory and ovulatory infertility in obese women.

\section{Obesity and assisted reproduction}

Obesity is associated with a lower chance of live birth after IVF/intracytoplasmic sperm injection and with an impaired response to ovarian stimulation.

Obesity reduces the likelihood that a woman will be accepted for assisted reproductive techniques (ART) treatment, particularly in a nationally funded situation.

\section{Obesity and obstetric care}

Overweight and obesity increases obstetric risks in a BMI-dependent manner. The risk of perinatal death and congenital abnormalities double in the obese mother.

\section{Managing obesity}

Weight loss improves menstrual regularity, ovulation and fertility, and should be promoted as an initial treatment option for obese women with infertility. Only 3-5\% weight loss is required.

Lifestyle modifications are the best way to achieve and sustain weight loss. These include sensible dieting, regular exercise, cognitive behavior therapy and a supportive group environment.

\section{Pharmacological \& surgical intervention}

Pharmacologic intervention with metformin is not superior to clomiphene to induce ovulation and pregnancy/live birth rate in obese PCOS women.

Surgical intervention should be reserved if other measures of weight reduction fail. Although effective, it must be used together with dietary modifications and behavioral changes.

\section{References}

[1]. Al-Azemi M, Omu FE, Omu AE (2004) the effect of obesity on the outcome of infertility management in women with polycystic ovary syndrome. Arch Gynaecol Obstet. 270: 2005-2010.

[2]. Azziz R (2006) How prevalent is metabolic syndrome in women with polycystic ovarian syndrome. Nat ClinPrac Endo Metab 2: 132-133.

[3]. Balarajan Y, Villamor E (2009) Nationally representative surveys show recent increases in the prevalence of overweight and obesity among women of reproductive age in Bangladesh, Nepal, and India. J Nutr. 139: 2139-2144.

[4]. Balen AH, Conway GS, Kaltsas G et al.: Polycystic ovary syndrome: the spectrum of the disorder in 1741 patients. Hum. Reprod. 10, 2107-2111 (1995).

[5]. Bjorge T, Engeland A, Tretli S et al.: Body size in relation to cancer of the uterine corpus in 1 million Norwegian women. Int. J. Cancer. 120(2), 378-383 (2007).

[6]. Ehrmann DA: Polycyctic ovary syndrome. N. Engl. J. Med. 352, 1223-1236 (2005).

[7]. Gouda J, Prusty RK (2014) Overweight and obesity among women by economic stratum in urban India. J Health PopulNutr. 32: 79-88.

[8]. Hamad G, Eid GM. The Female Patient: Pregnancy and Gynecological Issues in the Bariatric Surgery Patient. Chapter 38. Minimally Invasive Bariatric Surgery.

[9]. Hartz AJ, Rupley DC, Rimm AA: The association of girth measurements with disease in 32, 856 women. Am. J. Epidemiol. 119, 71-80 (1984).

[10]. Khan R, Dawlatly B, Chappatte O (2013) Pregnancy outcome following bariatric surgery. The Obstetrician \& Gynaecologist 15: 37-43.

[11]. Kirschner MA: Obesity, androgens, oestrogens, and cancer risk. Cancer Res. 42, 3281-3285 (1982). 
[12]. Kouris-Blazos A, Wahlqvist ML: Health economics of weight management: evidence and cost. Asia Pac. J. Clin. Nutr. 16(1), 329-338 (2007).

[13]. Moyad MA: Current methods used for defining, measuring, and treating obesity. Semin. Urol. Oncol. 19(4), 247-256 (2001).

[14]. Norman RJ, Masters SC, Hague W et al.: Metabolic approaches to the subclassification of polycyctic ovary syndrome. Fertil. Steril. 63, 329-335 (1995).

[15]. Pandey S, Pandey S, Maheshwari A, Bhattacharya S (2010) The impact of female obesity on the outcome of fertility treatment. J Hum Reprod Sci. 3: 62-67.

[16]. Pasquali R, Gambineri A, Pagotto U: The impact of obesity on reproduction in women with polycystic ovary syndrome. Br. J. Obstet. Gynaecol. 113, 1148-1159 (2006).

[17]. Pasquali R, Patton L, Gambineri A (2007) Obesity and infertility. Curr Opin Endocrinol Diabetes Obes. 14: 482-487.

[18]. Pasquali R: Obesity, fat distribution and infertility. Maturitas 54, 363-371 (2006).

[19]. Reeves GK, Pirie K, Beral V et al.: Cancer incidence and mortality in relation to body mass index in the Million Women Study: cohort study. BMJ 335(7630), 1134 (2007).

[20]. Siedell JC: The impact of obesity on health status: some implications for health care costs. Int. J. Obes. Relat. Metab. Disord. 19(6), S13-S16 (1995).

[21]. Stein IF, Leventhal ML: Amenorrhea associated with bilateral polycystic ovaries. Am. J. Obstet. Gynecol. 29, 181-191 (1934).

[22]. The Rotterdam ESHRE/ASRM-Sponsored PCOS consensus workshop group. Revised 2003 consensus on diagnostic criteria and long-term health risks related to polycyctic ovary syndrome. Hum. Reprod. 19, 41-47 (2004).

[23]. Wajchenberg BL: Subcutaneous and visceral adipose tissue: their relation to the metabolic syndrome. Endocr. Rev. 21, 697-738 (2000).

[24]. WebMD Health News Reviewed by Louise Chang, MD on December 11, 2007

[25]. Wolfe H: High prepregnancy body mass index- a maternal-fetal risk factor. N. Engl. J. Med. 338, 191-192 (1998).

[26]. World Health Organization: Obesity preventing and managing the global epidemic. Report of a WHO consultation on Obesity WHO/NUT/NCD 98.1. World Health Organization, Geneva, Switzerland (1997).

[27]. Zaadstra BM, Seidell JC, Van Noord PA et al.: Fat and female fecundity: prospective study of effect of body fat distribution on conception rates. Br. Med. J. 306, 484-487 (1993). 